\title{
2D DOA estimation for non-uniform L-shaped array via a successive Capon algorithm
}

\author{
Lv Weihua ${ }^{1,2}$, Zheng Wang ${ }^{1}$, Cheng Qianlin ${ }^{1}$, Zhang Xiaofei ${ }^{1,3, a}$ \\ 1. College of Electronic and Information Engineering, Nanjing University of Aeronautics and \\ Astronautics, Nanjing, China, 210016 \\ 2. Luoyang Optoelectro technology development center, Luoyang, China, 471000 \\ 3. National Mobile Communications Research Laboratory, Southeast University, China, 210096 \\ aEmail: zhangxiaofei@nuaa.edu.cn
}

Keywords: DOA, Successive, Capon, Non-uniform L-shaped Array

\begin{abstract}
Capon algorithm is a common algorithm for the estimating of direction of arrival (DOA). As it needs two-dimensional (2D) spectrum peak search which costs a tremendous computational complexity, in this paper, we propose a successive algorithm based on Capon algorithm for non-uniform L-shaped array to avoid the high computational complexity which only needs three one-dimensional spectrum peak search. The successive Capon algorithm has an acceptable performance and can obtain automatically paired 2D DOA estimation for L-shaped array. Extensive simulations have been conducted to verify the usefulness of the algorithm.
\end{abstract}

\section{Introduction}

The estimation of DOA is one of the fundamental problem in array signal processing and is very important for various engineering applications such as radar, sonar, wireless communication and medical imaging [1][2]. Among the various arrays like the parallel uniform linear array, the rectangular array and the circular array, L-shaped array which consists of two linear subarray connected orthogonally at one end of each array, is commonly used for 2D DOA estimation array as it features a simple structure and can obtain a relatively high estimation accuracy in practice. Thus 2D DOA estimation with L-shaped array has attract a great attention.

2D Capon algorithm exploits the suppression of the noise and interference coming from the direction of the non-sources. And it estimates the DOAs with the signal power invariance criterion. However, it needs 2D spectrum peak search which has a high computational complexity. Ref.[3] addresses a problem of blind 2D DOA estimation with L-shaped array. It links the 2D DOA estimation problem to the trilinear model and gets much better estimation performance. But it has a high computational complexity. In [4], Jiang proposed a reduced-dimension Capon algorithm for planar array which had a low computational complexity. In this paper, we propose the successive Capon algorithm which only needs three one-dimensional search to achieve the 2D DOA estimation and it also has a low computational complexity.

The reminder of this paper is organized as follows. In section 2, we present the data model. In section 3, we describe the successive Capon algorithm. Simulation results are presented in section 4 and we conclude this paper in section 5.

Notations: Lower-case (upper-case) boldface symbols denote vectors (matrices). $(\bullet)^{T},(\bullet)^{H},(\bullet)^{-1},(\bullet)^{+}$denote the transpose, the conjugate transpose, the inverse and the pseudo inverse, respectively. $E(\bullet)$ denotes the expectation operator.

\section{Data model}

We assume that there are $K$ uncorrelated narrowband far-field signals located at $\left\{\left(\theta_{k}, \phi_{k}\right) \mid k=1,2, \cdots, K\right\}$, where $\theta_{k}$ is the elevation angle $\phi_{k}$ is the azimuth angle of the kth 
source $(k=1,2, \cdots, K)$, impinging on the non-uniform L-shaped array equipped with $N$ sensors in $x$-direction and $M$ sensors in $z$-direction as shown in Fig.1. The sensors are located at $\mathbf{d} x=\left[d_{x 1}, d_{x 2}, \cdots, d_{x N}\right]$ in $x$-axis and $\mathbf{d} z=\left[d_{z 1}, d_{z 2}, \cdots, d_{z M}\right]$ in $z$-axis. The noise is additive independent identically distributed Gaussian with zero mean and variance $\sigma_{n}^{2}$, which is independent of signals.

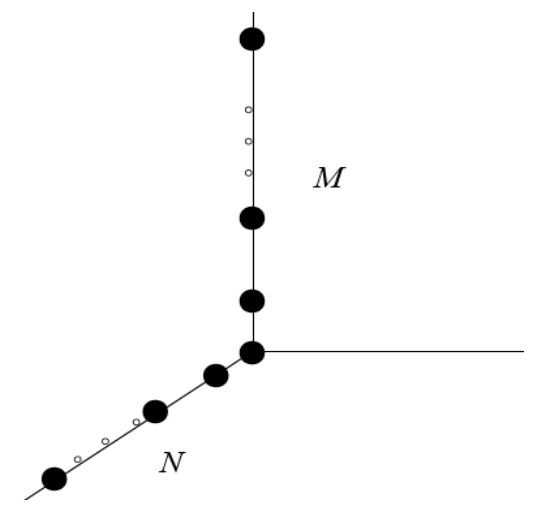

Fig.1 Non-uniform L-shaped array model

The received signal of each subarray can be represented as

$$
\begin{aligned}
& \mathbf{X}(t)=\mathbf{A}_{x} \mathbf{S}(t)+\mathbf{N}_{x}(t) . \\
& \mathbf{Z}(t)=\mathbf{A}_{z} \mathbf{S}(t)+\mathbf{N}_{z}(t) .
\end{aligned}
$$

where $\mathbf{S}(t) \in C^{K \times L}, \mathbf{N}_{x}(t) \in C^{N \times L}$ and $\mathbf{N}_{z}(t) \in C^{M \times L}$ ( $L$ denotes the number of snapshots).

The steering matrix $\mathbf{A}_{x} \in C^{N \times K}$ and $\mathbf{A}_{z} \in C^{M \times K}$ are given by $\mathbf{A}_{x}=\left[\mathbf{a}_{x}\left(\theta_{1}, \phi_{1}\right), \mathbf{a}_{x}\left(\theta_{2}, \phi_{2}\right), \cdots\right.$, $\left.\mathbf{a}_{x}\left(\theta_{K}, \phi_{K}\right)\right]$ and $\mathbf{A}_{z}=\left[\mathbf{a}_{z}\left(\theta_{1}, \phi_{1}\right), \mathbf{a}_{z}\left(\theta_{2}, \phi_{2}\right), \cdots, \mathbf{a}_{z}\left(\theta_{K}, \phi_{K}\right)\right]$, where $\mathbf{a}_{x}\left(\theta_{k}, \phi_{k}\right)=\left[e^{-j 2 \pi d_{x 1} \sin \theta_{k} \cos \phi_{k} / \lambda}\right.$, $\left.e^{-j 2 \pi d_{x 2} \sin \theta_{k} \cos \phi_{k} / \lambda}, \cdots, e^{-j 2 \pi d_{x N} \sin \theta_{k} \cos \phi_{k} / \lambda}\right]^{T}$ and $\mathbf{a}_{z}\left(\theta_{k}, \phi_{k}\right)=\left[e^{-j 2 \pi d_{z 1} \cos \theta_{k} / \lambda}, e^{-j 2 \pi d_{22} \cos \theta_{k} / \lambda}, \cdots, e^{-j 2 \pi d_{z M} \cos \theta_{k} / \lambda}\right]^{T}(\lambda$ is the wavelength).

The received signal of the L-shaped array can be represented as

$\mathbf{Y}(t)=\left[\begin{array}{l}\mathbf{X}(t) \\ \mathbf{Z}(t)\end{array}\right]=\left[\begin{array}{l}\mathbf{A}_{x} \\ \mathbf{A}_{z}\end{array}\right] \mathbf{S}+\left[\begin{array}{l}\mathbf{N}_{x} \\ \mathbf{N}_{z}\end{array}\right]$.

Then we can obtain the covariance matrix of the L-shaped array,

$\mathbf{R}_{y}=\mathbf{Y}(t) \mathbf{Y}^{H}(t) / L$

The spatial spectrum function of $2 \mathrm{D}$ Capon is given by

$$
f_{y}\left(\theta_{k}, \phi_{k}\right)=\frac{1}{\mathbf{a}^{H}\left(\theta_{k}, \phi_{k}\right) \mathbf{R}_{y}^{-1} \mathbf{a}\left(\theta_{k}, \phi_{k}\right)} \text {. }
$$

where $\mathbf{a}\left(\theta_{k}, \phi_{k}\right)=\left[\begin{array}{c}\mathbf{a}_{x}\left(\theta_{k}, \phi_{k}\right) \\ \mathbf{a}_{z}\left(\theta_{k}, \phi_{k}\right)\end{array}\right]$ and $\theta_{k} \in[0, \pi / 2], \phi_{k} \in[0, \pi]$. By 2D spectrum peak search which bring a tremendous computational complexity, we can obtain the estimations of elevation and azimuth angle.

\section{The successive Capon algorithm}

\subsection{The first one-dimensional search}

In this section, we utilize the subarray in the z-axis to implement the one-dimensional Capon algorithm so that we can get the estimation of the elevation angle $\hat{\theta}$. According to (2), we can get 
the covariance matrix of the subarray on the $z$-axis, in practice,

$$
\mathbf{R}_{z}=\mathbf{Z}(t) \mathbf{Z}^{H}(t) / L \text {. }
$$

The spatial spectrum function is given by

$$
f_{z}\left(\theta_{k}\right)=\frac{1}{\mathbf{a}^{H}\left(\theta_{k}\right) \mathbf{R}_{z}^{-1} \mathbf{a}\left(\theta_{k}\right)} \text {. }
$$

where $\mathbf{a}\left(\theta_{k}\right)=\left[e^{-j 2 \pi d_{z 1} \cos \theta_{k} / \lambda}, e^{-j 2 \pi d_{z 2} \cos \theta_{k} / \lambda}, \cdots, e^{-j 2 \pi d_{z M} \cos \theta_{k} / \lambda}\right]^{T}$. By one-dimensional search via $\theta$, we can obtain the initial estimation of elevation angle $\hat{\boldsymbol{\theta}}_{i n i}=\left[\hat{\theta}_{i 1}, \hat{\theta}_{i 2}, \cdots, \hat{\theta}_{i K}\right]$.

\subsection{The second one-dimensional search}

In this section, we utilize the L-shaped array to implement the second one-dimensional search with the initial estimation of elevation angle in 3.1.

The spatial spectrum function is given by

$$
f_{y}\left(\hat{\theta}_{i n}, \phi_{k}\right)=\frac{1}{\mathbf{a}^{H}\left(\hat{\theta}_{i n}, \phi_{k}\right) \mathbf{R}_{y}^{-1} \mathbf{a}\left(\hat{\theta}_{i n}, \phi_{k}\right)} \text {. }
$$

where $\mathbf{a}\left(\hat{\theta}_{i n}, \phi_{k}\right)=\left[\begin{array}{c}\mathbf{a}_{x}\left(\hat{\theta}_{i n}, \phi_{k}\right) \\ \mathbf{a}_{z}\left(\hat{\theta}_{i n}, \phi_{k}\right)\end{array}\right]$ and $\hat{\theta}_{i n} \in \hat{\boldsymbol{\theta}}_{i n i}$.

By one-dimensional search via $\phi_{k}$, we can get the estimation of azimuth angle $\hat{\boldsymbol{\Phi}}=\left[\hat{\phi}_{1}, \hat{\phi}_{2}, \cdots, \hat{\phi}_{K}\right]$.

\subsection{The third one-dimensional search}

In this section, we utilize the L-shaped array to implement the third one-dimensional search with the estimation of azimuth angle in 3.2. Then the spatial spectrum function should be rewritten as

$$
f_{y r}\left(\theta_{k}, \hat{\phi}_{n}\right)=\frac{1}{\mathbf{a}^{H}\left(\theta_{k}, \hat{\phi}_{n}\right) \mathbf{R}_{y}^{-1} \mathbf{a}\left(\theta_{k}, \hat{\phi}_{n}\right)} .
$$

where $\quad \mathbf{a}\left(\theta_{k}, \hat{\phi}_{n}\right)=\left[e^{-j 2 \pi d_{x 1} \sin \theta_{k} \cos \hat{\phi}_{n} / \lambda}, e^{-j 2 \pi d_{x 2} \sin \theta_{k} \cos \hat{\phi}_{n} / \lambda}, \cdots, e^{-j 2 \pi d_{x N} \sin \theta_{k} \cos \hat{\phi}_{n} / \lambda}\right]^{T} \quad$ and $\quad \hat{\phi}_{n} \in \hat{\mathbf{\Phi}}$. By one-dimensional search via $\theta$, we can get the second estimation of elevation angle $\hat{\boldsymbol{\theta}}_{\text {sec }}=\left[\hat{\theta}_{s 1}, \hat{\theta}_{s 2}, \cdots, \hat{\theta}_{s K}\right]$ which is more accurate than the initial ones and simulations will verify it in the following section.

\subsection{Complexity analysis}

The complexity of 2D Capon is $(M+N)^{2} L+(M+N)^{3}+l_{1} l_{2}\left[(M+N)^{2}+(M+N)\right]$ and the one of the proposed algorithm is $M^{3}+(M+N)^{3}+M^{2} K+(M+N)^{2} K+l_{1}\left(M^{2}+M\right)+\left(l_{1}+l_{2}\right)\left[(M+N)^{2}\right.$ $+(M+N)$ ], where $l_{1}$ is the search number of elevation angle and $l_{2}$ is the search number of azimuth angle. The complexity of the proposed algorithm is lower than that of the 2D Capon which is illustrated in Fig.2.

\section{Simulation results}

In this section, we illustrate the performance of the successive Capon algorithm for non-uniform L-shaped array. Suppose $K=2$ sources impinging on the array located at $\left(\theta_{1}, \phi_{1}\right)=\left(20^{\circ}, 45^{\circ}\right)$, $\left(\theta_{2}, \phi_{2}\right)=\left(40^{\circ}, 35^{\circ}\right)$. The root mean square error (RMSE) of the estimations is defined as the performance metric 


$$
R M S E=\sqrt{\frac{1}{S K} \sum_{s=1}^{S} \sum_{k=1}^{K}\left(\alpha_{k}-\hat{\alpha}_{k, s}\right)^{2}} .
$$

where $S$ denotes the times of Monte-Carlo simulations and $\hat{\alpha}_{k, s}$ is the estimation of the $k$ th angle $\alpha_{k}$ for the sth trial $(S=200)$.

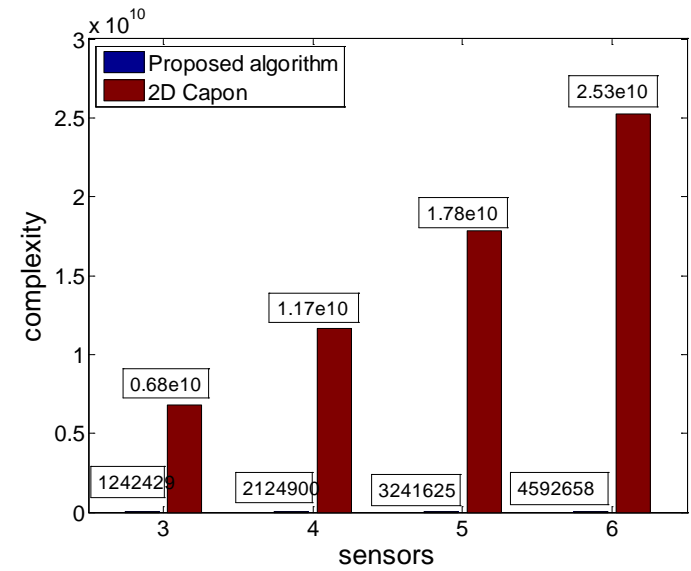

Fig.2 Comparison of computational complexity

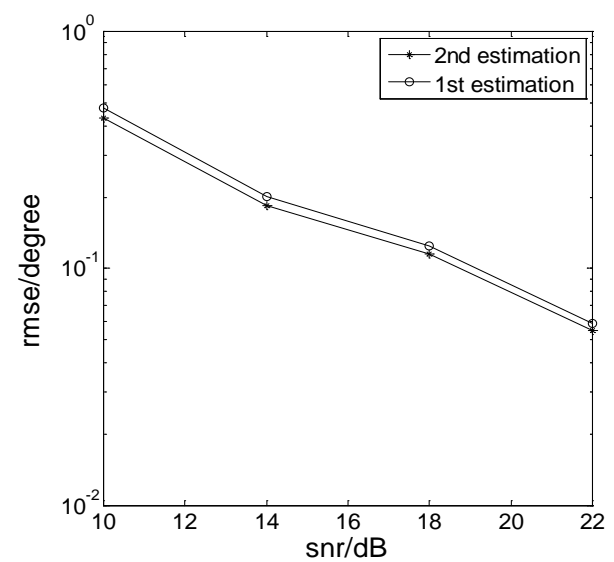

Fig.3 Comparison between the two estimations of elevation angles

In Fig.3, we compare the two estimations of elevation angle where $M=N=8, L=200$. It is indicated that the second estimations are more accurate than the first estimations. Then, we change the number of sensors as shown in Fig. 4 where $L=200$. It is clearly indicated that the performance of proposed algorithm is getting better and better with the number of the sensors increasing because of diversity gain. Finally, we change the number of snapshots $L$, where $M=N=8$ as shown in Fig.5. It is clearly indicated that the performance of the proposed algorithm is getting better and better with $L$ increasing because the larger the number of snapshots is, the more accurate the covariance matrix of the received data is.

\section{Summary}

In this paper, we propose a successive Capon algorithm for non-uniform L-shaped array. As it needs only three one-dimensional spectrum peak search which significantly reduces the computational complexity with an acceptable performance compared with 2D Capon algorithm.

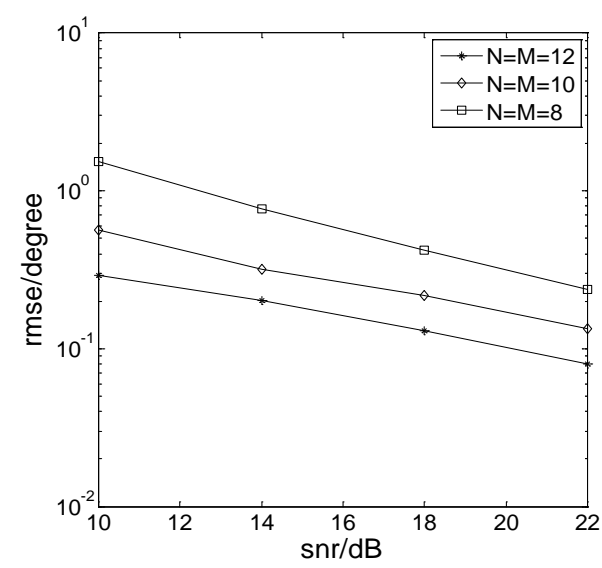

Fig.4 Performance of proposed algorithm with different number of sensors.

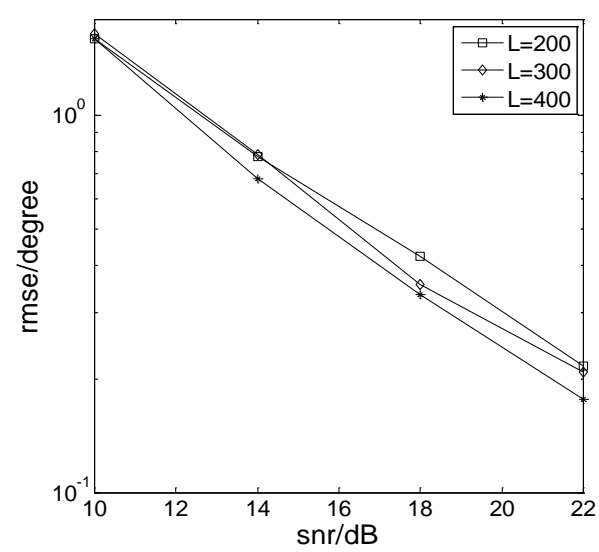

Fig.5 Performance of proposed algorithm with different snapshots. 


\section{Acknowledgments}

This work is supported by China NSF Grants (61371169, 61271327), the open research fund of

National Mobile Communications Research Laboratory, Southeast University (no.2015D03), Funding of Jiangsu Innovation Program for Graduate Education (KYLX15 0281), Funding for Outstanding Doctoral Dissertation in NUAA (BCXJ15-03), Qing Lan Project, and priority academic pro-gram development of Jiangsu high education institutions.

\section{References}

[1] Yang D S, Chen H, Shi S G. A near-field and high-resolution localization method of an under-water noise source based on a MUSIC algorithm [J]. Journal of Harbin Engineering University, vol.32, no.8, pp: 1019-1023, 2011.

[2] Lavate T B, Kokate V K, Sapkal A M. Performance analysis of MUSIC and ESPRIT DOA estimation algorithms for adaptive array smart antenna in mobile communication [C]. 2010 Second International Conference on Computer and Network Technology (ICCNT), pp: 308-311, 2010.

[3] Zhang X, Li J, Xu L. Novel two-dimensional DOA estimation with L-shaped array [J]. Journal on Advances in Signal Processing, vol.2011, no.1, pp: 1-7, 2011.

[4] Jiang Chi, Zhang Xiaofei, Zhang Licen. Two-Dimensional DOA Estimation for Planar Array via Reduced-Dimension Capon [J]. Journal of Applied Sciences, vol.33, no.2, pp: 167-177, 2015.

[5] Wu Y T, Liao G S, So H C. A fast algorithm for 2-D direction-of-arrival estimation [J]. Signal Process, vol.83, no.8, pp: 1827-1831, 2003.

[6] Zhang Xiaofei, Wang Fei, Chen Weihua. The theory and application of array signal processing. Beijing: National Defence Industry Press, 2013.

[7] Wang G, Xin J, Zheng N \& Sano. A Computationally efficient subspace-based method for two-dimensional direction estimation with L-shaped array. IEEE Transactions on Signal Processing, vol.59, no.7, pp: 3197-3212, 2011.

[8] Chen J, Wang S X, Wei X L. New method for estimating two-dimensional direction of arrival based on L-shape array [J]. Journal of Jilin University, vol.36, no.4, pp: 590-593, 2006. 